Martin Grader, Zhiyao Yin, Klaus Peter Geigle, Peter Gerlinger, Influence of flow field dynamics on soot evolution in an aero-engine model combustor,

Proceedings of the Combustion Institute 38 (2021) 6421-6429

The original publication is available at www.elsevier.com

https://doi.org/10.1016/j.proci.2020.05.019

(C) <2021>. This manuscript version is made available under the CC-BY-NC-ND

4.0 license http://creativecommons.org/licenses/by-nc-nd/4.0/ 


\title{
Influence of flow field dynamics on soot evolution in an aero-engine model combustor
}

\author{
Martin Grader,*, Zhiyao Yin ${ }^{\mathrm{a}}$, Klaus Peter Geigle ${ }^{\mathrm{a}}$, Peter Gerlinger ${ }^{\mathrm{a}}$ \\ ${ }^{a}$ German Aerospace Center (DLR), Institute of Combustion Technology, Pfaffenwaldring \\ 38-40, 70569 Stuttgart, Germany
}

\begin{abstract}
Large-Eddy Simulation of a sooting aero-engine model combustor is analyzed by means of proper orthogonal decomposition (POD) to investigate the influence of flow field dynamics on soot evolution. Since classical POD fails to extract the low-frequency intermittent dynamics of scalars such as soot volume fraction, the recently-introduced multiresolution POD (MRPOD) is deployed to treat the LES results instead. MRPOD essentially provides additional dynamic rankings of energetic POD modes via bandpass filters constructed using wavelet packet transform (WPT). In the investigated combustor major dynamics identified in both velocity and scalar fields include a precessing vortex core (PVC) at about $430 \mathrm{~Hz}$ and low-frequency dynamics (LFD) at below $100 \mathrm{~Hz}$. Low-frequency dynamics of velocity are present in the whole combustor and affect the secondary air injection and recirculation. While both dynamics are found to influence soot volume fraction, the PVC impact is much smaller compared to the LFD impact.
\end{abstract}

Keywords:

\footnotetext{
*Corresponding author: Martin Grader

Email address: martin.grader@dlr.de (Martin Grader)
} 
Large-Eddy Simulation, Multiresolution Proper Orthogonal Decomposition, Soot, Gas Turbine

\section{Introduction}

Soot emissions from aero-engine combustors have received continuous attention over the last decades due to their adverse effects an human health, engine efficiency and climate. Significant improvement in the understanding of soot emissions under engine-like conditions has not only been achieved by experiments, but also by high fidelity computational fluid dynamics (CFD). Notably growing computational power enables CFD to resolve transient reactive fluid motion by Large-Eddy Simulation (LES), which is not only useful for engineering applications but also contributes to the understanding of physical phenomena in complex turbulent flows. A wide variety of coherent and transient dynamics can coexist in aero-engine combustors, such as thermoacoustics, a precessing vortex core (PVC), and large-scale fluctuations. However, the impacts of these well-known dynamics on soot evolution have not yet been extensively analyzed.

The sooting aero-engine model combustor first investigated by Geigle et al. [1] is an ideal test case. Due to high soot intermittency, it is well suited for analyzing the influence of the combustor dynamics on soot evolution. Eberle et al. [2] observed a strong impact of the PVC on mixing of the injected fuel and air with recirculated hot gases in the inner recirculation zone (IRZ), where most of the soot formation occurs. On the other hand, LES studies by

Chong et al. [3] showed a strong influence of low-frequency large-scale fluctuations on soot. In a combined experimental and numerical study, Stöhr et 
al. [4] supported this finding. It is shown that an intermittent low-frequency recirculation of lean burned gas influences the residence time of fuel-rich, partially soot-containing pockets in the IRZ.

Based on these observations, a detailed analysis of a time-resolved finite-rate chemistry LES data is performed. The testcase is the same sooting flame and for analysis a recently-introduced variant of proper orthogonal decomposition (POD): multiresolution POD (MRPOD) [5] is employed. The major shortcoming of conventional POD is the lack of dynamic ranking, such that multiple dynamic components with different characteristic frequencies could be lumped into a single mode, hindering unambiguous interpretations of the results [6]. MRPOD, on the other hand, incorporates the concept of waveletbased multiresolution analysis (MRA) into a snapshot POD to provide additional dynamic ranking for the isolation of various frequency components during modal decomposition. Unlike a purely dynamics-promoting modal analysis such as dynamic mode decomposition (DMD) [7], MRPOD is capable of preserving non-periodic fluctuations and resolving intermittencies. This is a critical feature necessary to correctly capture the sporadic soot evolution in the target flame. Specifically, this work employs a maximumoverlap discrete packet wavelet transform (MODPWT) in the MRPOD to isolate PVC from low-frequency dynamics (LFD), such that their influences on the flow field as well as on soot volume fraction $\left(f_{\mathrm{v}}\right)$ can be separately evaluated. Beside POD-based methods the cluster-based reduced-order modeling (CROM) method $[8,9]$ with its probabilistic nature, may also prove appropriate at treating the nonlinear LFD. 


\section{Experimental and numerical setup}

Figure 1 shows the investigated aero-engine model combustor. The pri-

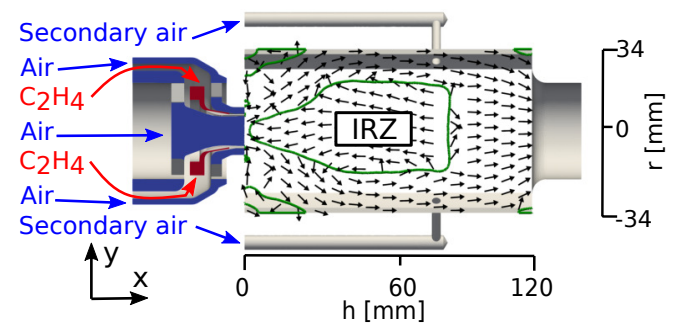

Figure 1: Schematic of the aero-engine model combustor. Iso-contours of $\overline{\mathrm{v}}_{\mathrm{x}}=0 \mathrm{~m} / \mathrm{s}$ indicate recirculation, arrows the velocity field.

mary injector consists of two concentric, swirled airflows, into which ethylene is injected co-axially via 64 rectangular channels. A secondary air injection at $\mathrm{h}=80 \mathrm{~mm}$ mimics the rich quench lean (RQL) concept of industrial aeroengine combustors. The reference operating conditions as defined in [1] are chosen in this work. The combustor is operated at three bar and at a primary equivalence ratio of $\Phi_{p}=1.2$ and a global equivalence ratio of $\Phi_{g}=0.86$, including secondary air. The fuel mass flow is $\dot{\mathrm{m}}_{\mathrm{C}_{2} \mathrm{H}_{4}}=0.83 \mathrm{~g} / \mathrm{s}$, the primary inner and outer air injection mass flow is $\dot{\mathrm{m}}_{\text {air }_{\mathrm{i}}}=3.03 \mathrm{~g} / \mathrm{s}$ and $\dot{\mathrm{m}}_{\text {airo }_{\mathrm{o}}}=7.08 \mathrm{~g} / \mathrm{s}$, respectively and the secondary air mass flow is $\dot{\mathrm{m}}_{\text {air }}=4.04 \mathrm{~g} / \mathrm{s}$. Fuel and air are injected with a temperature of $293 \mathrm{~K}$.

The LES has been performed with the DLR in-house code THETA on an unstructured 38-million-volumes grid. The incompressible solver uses a projection method for pressure-velocity coupling and a second-order discretization in space and time. The influence of unresolved subgrid turbulence is modeled by the WALE model [10]. Turbulence-chemistry interaction is accounted 
for by an assumed-PDF approach, where sub-grid variances are calculated with a scale similarity approach [11]. Assuming statistical independence, a Gaussian-shaped temperature PDF, a joint beta-PDF for species [12, 13] and a double-delta PDF for soot [14] are applied. Due to the chosen finite-rate chemistry approach, no presumptions about the combustion regime are required. A well tested gas-phase mechanism for ethylene combustion under sooting conditions [15] with 43 gas-phase species [16] is applied and differential diffusion is considered. The combination of gas phase mechanism and sectional models for polycyclic aromatic hydrocarbons (PAHs) and soot was developed by Eberle et al. [17, 18]. It has been validated for a large number of laminar premixed and non-premixed flames as well as for turbulent combustion. This model has been significantly improved by the introduction of PAH-radicals [17]. PAHs and their radicals cover the range between $100 \mathrm{~g} / \mathrm{mol}$ and $800 \mathrm{~g} / \mathrm{mol}$. Soot nucleation is based on PAH collision reactions involving the last PAH section. By assuming spherical soot particles in the first, adjacent soot section, a realistic nascent soot particle diameter of $1.28 \mathrm{~nm}$ is achieved. As the three PAH sections, all soot sections sizes are scaled by a factor of two with the first section covering particles of $800 \mathrm{~g} / \mathrm{mol}$ to $1600 \mathrm{~g} / \mathrm{mol}$. The sectional soot model consists of 30 sections including both spherical soot particles and aggregates. For details concerning the treatment of aggregation, see [18]. Solving all species, PAHs, and soot transport equations in a fully coupled way guarantees that feedback effects between gas phase and soot are captured inherently correct. Further details concerning the setup of the present LES and the aero-engine model combustor [1] are given in [19]. Every CFD analysis depends on accurate simulation 
models to predict the underlying physics correctly. To evaluate the quality of the approaches used in this work, the reader is referred to Grader et al. [19] where an almost identical simulation revealed a good overall agreement with measured first and second order moments. Instantaneous fields close to the primary injector are even in excellent agreement with the experiment [4]. The LES of this work differs from the former one by considering measured window temperature profiles [20]. This, however, causes no major difference compared to the previous LES calculation $[19,4]$. The final LES dataset consists of 805 result files or snapshots, sampled with a physical frequency of $4000 \mathrm{~Hz}$ over $201.25 \mathrm{~ms}$ sampling time.

\section{Fundamentals of MRPOD}

In principle, MRPOD extends the capability of the conventional snapshot POD [21] by introducing an additional step of wavelet-based MRA to achieve spectral isolation of target frequency components. Detailed derivations and characterizations of MRPOD are provided in [5]. Only the implementations specific to this work are summarized below.

For a variable $\alpha(x, t)$ (such as a velocity component or a scalar) from LES,

discretized in space $x \in \mathbb{R}^{M}$ and time $t \in \mathbb{R}^{N}$, the general goal of POD is to express $\alpha(x, t)$ as a linear combination of the so-called spatial modes $\phi_{l}(\alpha, x)$ multiplied by their corresponding temporal coefficients $a_{l}(\alpha, t)$ :

$$
\alpha^{\prime}(x, t)=\sum_{l=1}^{N} a_{l}(\alpha, t) \phi_{l}(\alpha, x) .
$$

Reynolds decomposition notation $\left(\alpha=\bar{\alpha}+\alpha^{\prime}\right)$ is applied to point out that the ensemble average of the dataset is subtracted from $\alpha(x, t)$ as tradition- 
ally done in POD. In order to improve readability, the inverted comma is only mentioned when Eq. (1) is used for reconstruction. In POD, the spatial modes are generated and ranked solely based on their energy $\left(E_{l}(\alpha)\right)$ contributions to $\alpha(x, t)$, where $E_{l}(\alpha)=\left\|a_{l}(\alpha)\right\|$. This process often groups multiple frequency components in one mode and can fail at capturing dynamically important but less energetic structures in the flow field. MRPOD overcomes this drawback by performing POD on various sub-scales of $\alpha(x, t)$, each of which correlates to a unique bandpass in the spectral domain and can be designed to encompass only a single frequency component, leading to simultaneous dynamic and energy decomposition of $\alpha(x, t)$. For MRPOD of a specific variable from the LES dataset, the following steps are carried out in this work:

1. $\alpha(x, t)$ is arranged in a matrix form $\mathbf{B}(\alpha) \in \mathbb{R}^{M \times N}$, with M representing the number of grid points and $\mathrm{N}$ the number of time steps (snapshots).

2. The cross-correlation matrix $\mathbf{K}(\alpha) \in \mathbb{R}^{N \times N}$ is constructed as:

$$
\mathbf{K}(\alpha)=\mathbf{B}(\alpha)^{\mathrm{T}} \mathbf{B}(\alpha)
$$

3. $\mathbf{K}(\alpha)$ is decomposed using two-dimensional MODWPT based on a Daubechies least asymmetric wavelet [22] with 16 basic elements, a decomposition level of 5 and reconstructed within various well-defined spectral bandpasses $\mathcal{I}$.

4. The eigenvalue problems of the resulting $\mathbf{K}(\alpha)_{\mathcal{I}}$ are solved to obtain the temporal coefficients $a_{l}(\alpha, t)$ and the spatial modes $\phi_{l}(\alpha, x)$ within the specified bandpasses $\mathcal{I}$.

As can be seen, steps 1,2 and 4 (with an unfiltered $\mathbf{K}(\alpha)$ ) constitute a con- 
ventional snapshot POD. Step 3 is essentially an MRA of $\alpha(x, t)$ in the time domain, which is equivalent to filtering $\alpha(x, t)$ at each individual grid point $x$ [5], albeit much less efficiently due to $\mathrm{M} \gg \mathrm{N}$ in LES. Additionally, $\mathbf{K}(\alpha)_{\mathcal{I}}$ could also be constructed using Fourier Impulse Response (FIR) based bandpass filters, as demonstrated in [5]. Several bandpasses have been designed to isolate prominent dynamics including LFD, PVC and higher-order harmonics of PVC in the flow field. This work focuses on LFD and PVC, which are obtained by setting the (nominal) bandpass to $\mathcal{I}_{\mathrm{LFD}}=[0,125] \mathrm{Hz}$ and $\mathcal{I}_{\mathrm{PVC}}=[375,625] \mathrm{Hz}$ during step 3, respectively. The sampling frequency of $4000 \mathrm{~Hz}$ guarantees a sufficient temporal resolution.

MRPOD has demonstrated remarkable effectiveness when treating non-periodic and highly intermittent fluctuations in a bi-stable turbulent swirl flame [5]. Such advantage over POD is also found when analyzing the LES results in this work, especially for capturing sporadic events in soot evolution from a fairly small sample size of 805 snapshots. Figure 2 compares the temporal coefficients of the most energetic pair of spatial modes $a_{1}, a_{2}$ extracted from $\mathrm{f}_{\mathrm{v}}$ by POD and MRPOD. They are expected to represent the PVC since it is the most energetic motion in the combustor. A Fast Fourier-Transformation (FFT) of the POD-based time coefficient $a_{1}\left(\mathrm{f}_{\mathrm{v}}\right)$ is shown in Fig. 2a. $a_{2}\left(\mathrm{f}_{\mathrm{v}}\right)$ exhibits a nearly identical spectral profile and is therefore omitted here. As can be seen, $a_{1}\left(f_{\mathrm{v}}\right)$ contains a notable peak centered around $430 \mathrm{~Hz}$, which has been linked previously to PVC [4]. In addition, a low-frequency footprint at below $100 \mathrm{~Hz}$ displays a higher amplitude and is lumped into the same mode with PVC by POD. A coherent structure such as PVC is expected to appear in a pair of POD modes that have nearly identical energy contribu- 

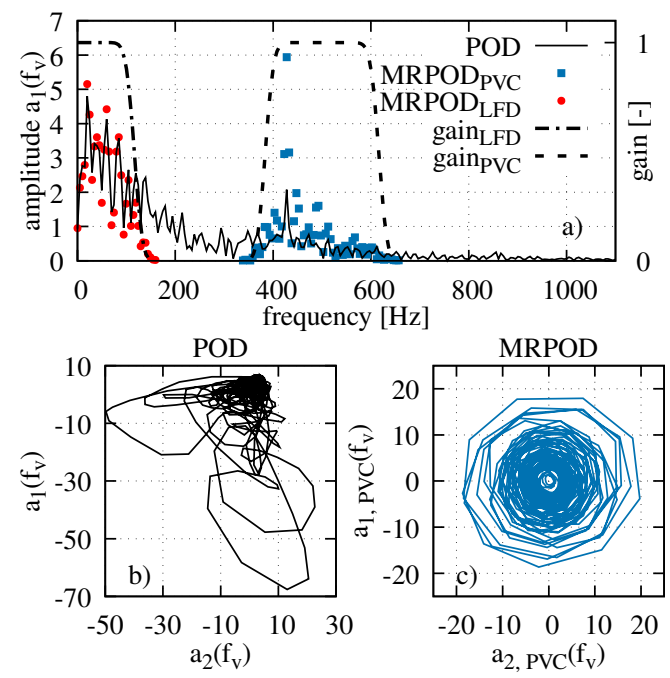

Figure 2: a) POD and MRPOD temporal coefficient spectra of $f_{v}$ and gain functions of the MRPOD bandpass filters; b) and c) correlation of PVC associated temporal coefficients $a_{1}$ and $a_{2}$ of $\mathrm{f}_{\mathrm{v}}$, for POD and MRPOD.

tions as well as sinusoidal temporal coefficients with a relative phase shift of $\pi / 2$ [23]. Therefore, a phase portrait of the temporal coefficients would reveal a near circular correlation. This is however not the case with $a_{1}\left(\mathrm{f}_{\mathrm{v}}\right)$ and $a_{2}\left(\mathrm{f}_{\mathrm{v}}\right)$ from POD, even though they seem to be associated with PVC, as shown in Fig. 2b. This rather chaotic picture is likely caused by the interference of the energetic low-frequency fluctuation that is indistinguishable to PVC for the energy-ranking mechanism of POD. With the aforementioned bandpasses of $\mathcal{I}_{\mathrm{PVC}}$ and $\mathcal{I}_{\mathrm{LFD}}$, MRPOD is able to isolate PVC from LFD, as shown in the FFT of $a_{1, \mathrm{LFD}}\left(\mathrm{f}_{\mathrm{v}}\right)$ and $a_{1, \mathrm{PVC}}\left(\mathrm{f}_{\mathrm{v}}\right)$ in Fig. 2a. Notably, the PVC amplitude is also higher in the case of MRPOD since POD failed to extract the PVC in the $\mathrm{f}_{\mathrm{v}}$ field and falsely distributed the PVC motion into 4 modes (not shown). MRPOD correctly ranked the PVC into 2 modes, which raises 
the amplitude of each mode. The temporal correlation between $a_{1, \mathrm{PVC}}\left(\mathrm{f}_{\mathrm{v}}\right)$ and $a_{2, \mathrm{PVC}}\left(\mathrm{f}_{\mathrm{v}}\right)$, plotted in Fig. 2c, clearly demonstrates the typical behavior of a mode pair, with large fluctuating amplitude (radius of the circle) indicating the extremely unsteady nature of soot evolution.

\section{LFD and PVC in the flow field}

The modal representations of the major dynamics in the flow field, LFD and PVC, are discussed in this section. Spatial modes and temporal coefficients are obtained by performing MRPOD on the axial velocity $\left(\mathrm{v}_{\mathrm{x}}\right)$ within $\mathcal{I}_{\text {LFD }}$ and $\mathcal{I}_{\text {PVC }}$. The energy contributions (both relative and cumulative) of the MRPOD modes are plotted in Fig. 3a-b for LFD and PVC, respectively. For PVC, a predominant mode pair $(l=1,2)$ can be readily identified. They
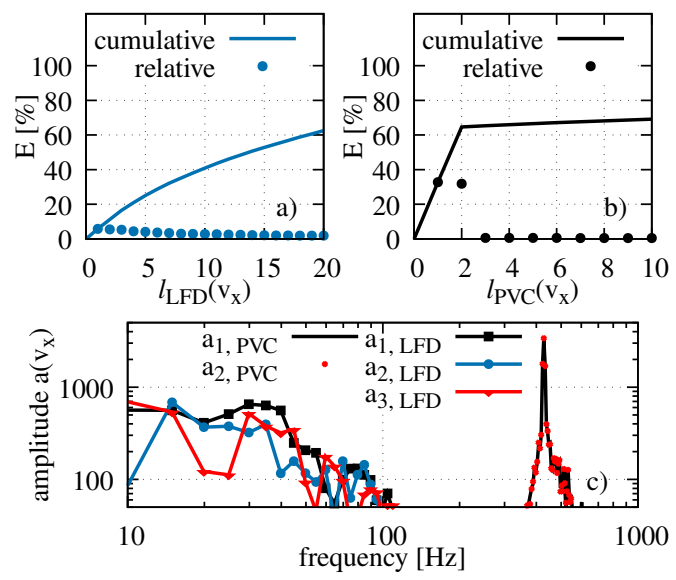

Figure 3: MRPOD of the axial velocity $\mathrm{v}_{\mathrm{x}}$ : Energy contributions of the MRPOD modes for a) LFD and b) PVC; c) FFT of the most energetic modes in LFD and PVC.

contribute to nearly $60 \%$ of the total energy contained within $\mathcal{I}_{\mathrm{PVC}}$ in $\mathrm{v}_{\mathrm{x}}$. On the other hand, there is no clear mode pair in the case of LFD, which 
shows about $60 \%$ of the kinetic energy to be relatively evenly distributed among the first 20 modes. From the FFT of the temporal coefficients of the strongest modes shown in Fig. 3c, it can be seen that while the two PVC modes exhibit the identical, well-defined frequency at about $430 \mathrm{~Hz}$, LFD modes are characterized by broadband features that do not seem to have any characteristic frequency. These results demonstrate that, while PVC is clearly a periodic, coherent fluctuation in the flow field, LFD is composed of a group of incoherent, non-periodic, loosely-correlated low-frequency dynamics.

The spatial influences of LFD and PVC in the flow field can be visualized and understood with the aid of their corresponding spatial MRPOD modes, as shown in Fig. 4. For demonstrative purpose $\phi_{2, \mathrm{LFD}}\left(\mathrm{v}_{\mathrm{x}}\right)$ and $\phi_{1, \mathrm{PVC}}\left(\mathrm{v}_{\mathrm{x}}\right)$

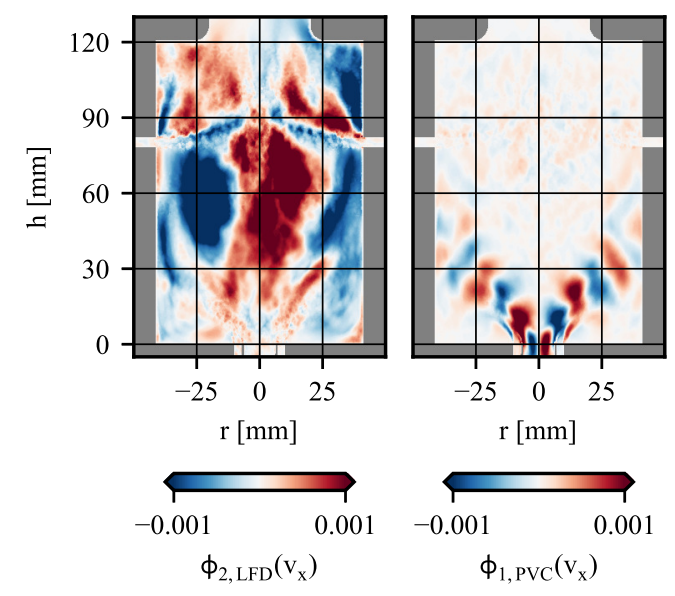

Figure 4: Spatial modes of axial velocity $\mathrm{v}_{\mathrm{x}}$ for LFD bandpass (left) and PVC bandpass (right) on a cross section through the combustor, including the secondary air injection.

are plotted on a cross section through the combustor, which includes the secondary air injection at $\mathrm{h}=80 \mathrm{~mm} . \mathrm{v}_{\mathrm{x}}$ is adopted here to emphasize the 
effect of LFD on the backflow. $\phi_{2, \mathrm{LFD}}\left(\mathrm{v}_{\mathrm{x}}\right)$ is chosen due to its relevance for further analysis. Other LFD modes show similar spatial distributions. While PVC influences the flow field primarily close to the injector along the shear layer between IRZ and the incoming flow, the LFD mode impacts the flow field in the entire combustor. In contrast to the PVC mode with its typical helical structure, evident from the "zig-zag" distribution of local maxima and minima, the shape of the LFD mode is more complex. High amplitude of $\phi_{2, \mathrm{LFD}}\left(\mathrm{v}_{\mathrm{x}}\right)$ along the centerline of the combustor indicates a low-frequency alternation of the backflow velocity in the IRZ and hence of the amount of recirculated gas. This is seemingly coupled to fluctuations of the secondary air injection as well as to the inlet flow (albeit weaker). The former can be recognized as a jet-flapping like motion as observed in confined jet-stabilized combustors [24]. The latter resembles the slow-varying shift mode commonly found in swirling flows [25].

\section{Influence of PVC on soot}

Figure 5 investigates the influence of the flow field PVC on computed soot-related quantities including a) mixture fraction Z , b) soot volume fraction $\left.f_{v}, c\right)$ mass fraction of the first PAH section $\mathrm{Y}_{\mathrm{PAH}_{0}}(\mathrm{PAHs}$ of 100 to 200 $\mathrm{g} / \mathrm{mol}$ ) and d) soot nucleation source term $\dot{\omega}_{\text {nuc }}$, by correlating their PVCrepresenting temporal coefficients $a_{1, \mathrm{PVC}}\left(\mathrm{Z} ; \mathrm{f}_{\mathrm{v}} ; \mathrm{PAH}_{0} ; \dot{\omega}_{\text {nuc }}\right)$ to $a_{1, \mathrm{PVC}}\left(\mathrm{v}_{\mathrm{x}}\right)$, analogous to the phase portraits in Fig. 2b-c. The interpretation of the phase

portraits is based on the following considerations: When the influence of PVC on $\mathrm{Z}, \mathrm{f}_{\mathrm{v}}, \mathrm{Y}_{\mathrm{PAH}_{0}}$ and $\dot{\omega}_{\text {nuc }}$ is in perfect synchronization with the vortex core movement in the flow field, one would expect either a "diagonal straight 

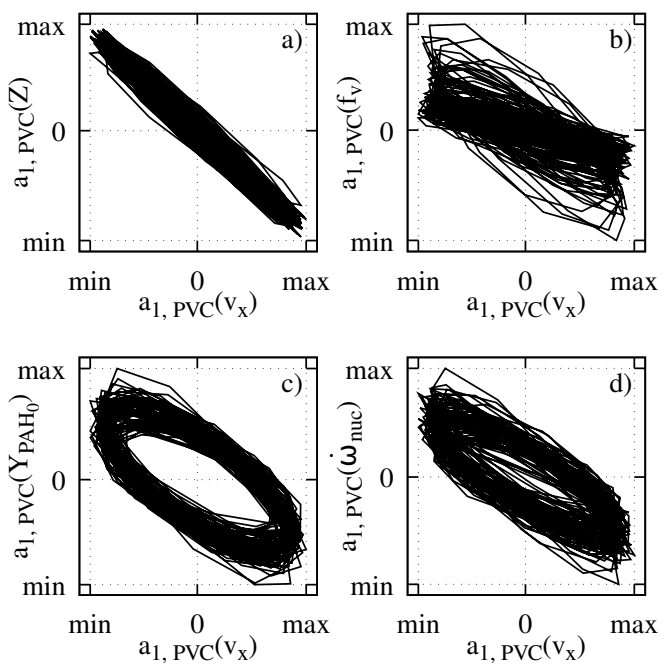

Figure 5: Correlation of $a_{1, \mathrm{PVC}}$ of axial velocity $\mathrm{v}_{\mathrm{x}}$ with a) mixture fraction $\mathrm{Z}$, b) soot volume fraction $\left.\mathrm{f}_{\mathrm{v}}, \mathrm{c}\right) \mathrm{PAH}_{0}$ mass fraction $\mathrm{Y}_{\mathrm{PAH}_{0}}$ and d) soot nucleation source term $\dot{\omega}_{\text {nuc }}$, respectively.

line" $(0 / \pi$ phase shift $)$ or a circular correlation $( \pm \pi / 2$ phase shift $)$ in the phase portrait such as in Fig. 2c. On the other hand, an elliptical phase portrait (arbitrary phase shift) would indicate that the flow field vortex core dynamics and its impact on the scalar are not concurrent in time. While the former case points to a pure mixing/displacement effect of PVC, the latter case suggests a PVC-induced change in local chemistry, resulting in a time lag relative to the vortex core motion.

A perfect synchronization is found between $a_{1, \mathrm{PVC}}\left(\mathrm{v}_{\mathbf{x}}\right)$ and $a_{1, \mathrm{PVC}}(\mathrm{Z})$ as shown in Fig. 5a. This is expected since the PVC enhances mixing and directly modifies Z. The same correlation is found between temperature and axial velocity (not shown). The PVC influence on soot evolution, represented by $f_{v}$, paints a more complicated picture, as shown in the phase portrait between $a_{1, \mathrm{PVC}}\left(\mathrm{f}_{\mathrm{v}}\right)$ and $a_{1, \mathrm{PVC}}\left(\mathrm{v}_{\mathrm{x}}\right)$ in Fig. 5b. Changing amplitudes of $a_{1, \mathrm{PVC}}\left(\mathrm{f}_{\mathrm{v}}\right)$ make 
the detection of a unique phase shift hardly possible. However, the elliptical shape of the phase portrait close to minimum and maximum of $a_{1, \mathrm{PVC}}\left(\mathrm{f}_{\mathrm{v}}\right)$ indicate the existence of a time delay between soot evolution and PVC. Since the phase portrait also passes the origin, synchronized motion with the flow field PVC dynamics is present, too. This implies that the influence of PVC on soot evolution is likely a combination of both time-delayed chemistry and instantaneous physical displacement of soot filaments. Further insight is gained by analyzing the correlation of PVC on soot precursors $\left(\mathrm{Y}_{\mathrm{PAH}_{0}}\right)$ and soot source terms $\left(\dot{\omega}_{\text {nuc }}\right)$ in Fig. 5c and Fig. 5d, respectively. $\dot{\omega}_{\text {nuc }}$ is chosen since it is not directly coupled to $\mathrm{f}_{\mathrm{v}}$. Soot growth and oxidation in turn are dependent on $f_{\mathrm{v}}$ and phase portraits are similar to Fig. 5b (not shown). Unlike in the cases of $\mathrm{Z}$ and $\mathrm{f}_{\mathrm{v}}$, a clear elliptical phase portrait can be established between $a_{1, \mathrm{PVC}}\left(\mathrm{Y}_{\mathrm{PAH}_{0}}\right)$ and $a_{1, \mathrm{PVC}}\left(\mathrm{v}_{\mathrm{x}}\right)$. This demonstrates that as the vortex core moves through the flow field, its influence on PAH is notably time-delayed, likely due to the slow PAH chemistry. On the other hand, a much smaller but still recognizable time delay (flatter elliptical phase portrait) can be observed between $a_{1, \mathrm{PVC}}\left(\dot{\omega}_{\text {nuc }}\right)$ and $a_{1, \mathrm{PVC}}\left(\mathrm{v}_{\mathrm{x}}\right)$, probably a result of short lifetime of PAH radicals, which are necessary for soot nucleation.

The PVC influence demonstrated above can be better understood with spatial visualization by taking advantage of the 3D LES results. For this purpose, reduced-order reconstructions based on only the PVC mode pair $\phi_{1, \mathrm{PVC}}$ and $\phi_{2, \mathrm{PVC}}$ and Eq. (1) are carried out for both $\mathrm{v}_{\mathrm{x}}$ and $\mathrm{Y}_{\mathrm{PAH}_{0}}$. Figure 6 shows overlaid streamlines and iso-contours of the reconstructed $\mathrm{v}_{\mathrm{x}, \mathrm{PVC}}^{\prime}$ and $\mathrm{Y}_{\mathrm{PAH}_{0}, \mathrm{PVC}}^{\prime}$ at a representative time instance. The streamlines reveal the position of the PVC vortices in the inner shear layer between primary injection 


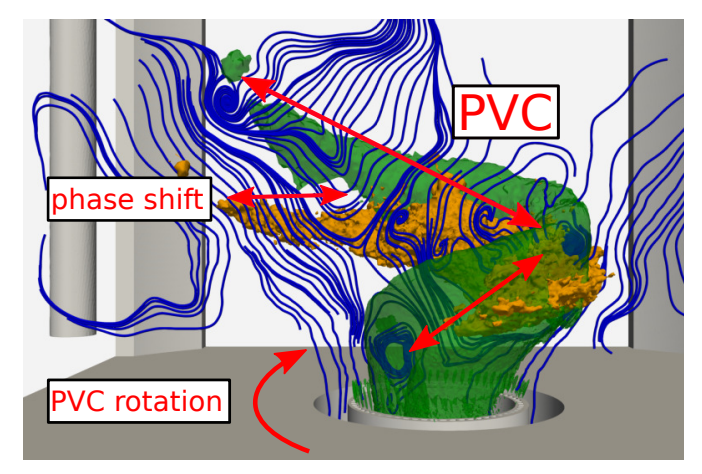

Figure 6: Instantaneous flow field on a cross section (blue streamlines) and reconstruction of $\mathrm{v}_{\mathrm{x}, \mathrm{PVC}}^{\prime}$ (green iso-contour $\mathrm{v}_{x, \mathrm{PVC}}^{\prime}=-3 \mathrm{~m} / \mathrm{s}$ ) and $\mathrm{Y}_{\mathrm{PAH}_{0}, \mathrm{PVC}}^{\prime}$ (orange iso-contour $\left.\mathrm{Y}_{\mathrm{PAH}_{0}, \mathrm{PVC}}^{\prime}=2.5 e-6\right)$ close to the primary injector.

and IRZ. The helical structure of the reconstructed velocity originates from the inner air nozzle, revealing the spiral evolution of the vortex core. PAH is mainly found in the IRZ, which causes the reconstructed $\mathrm{Y}_{\mathrm{PAH}_{0}, \mathrm{PVC}}^{\prime}$ field to appear downstream of the injector. The time-delayed response of PAH to PVC, shown in Fig. 5c is also present here, expressed by an offset in counter-rotation direction between $\mathrm{Y}_{\mathrm{PAH}_{0}, \mathrm{PVC}}^{\prime}$ and $\mathrm{v}_{\mathrm{x}, \mathrm{PVC}}^{\prime}$.

\section{Influence of low-frequency dynamics on soot}

As in the case of PVC, LFD exert influences on soot-related quantities as well, which are found to be coupled with the PVC influence in certain cases. This is demonstrated in Figure 7, where the influence of LFD and PVC on Z and $\mathrm{f}_{\mathrm{v}}$ are compared. Temporal evolution of $a_{1, \mathrm{LFD}}(\mathrm{Z})$ and $a_{1, \mathrm{PVC}}(\mathrm{Z})$ is shown in Fig. 7a. The sinusoidal signal of the PVC mode exhibits almost constant amplitude over time, seemingly not affected by the fluctuations induced by LFD. Temporal coefficients of $f_{v}$, shown in Fig. 7b, reveal a different behav- 


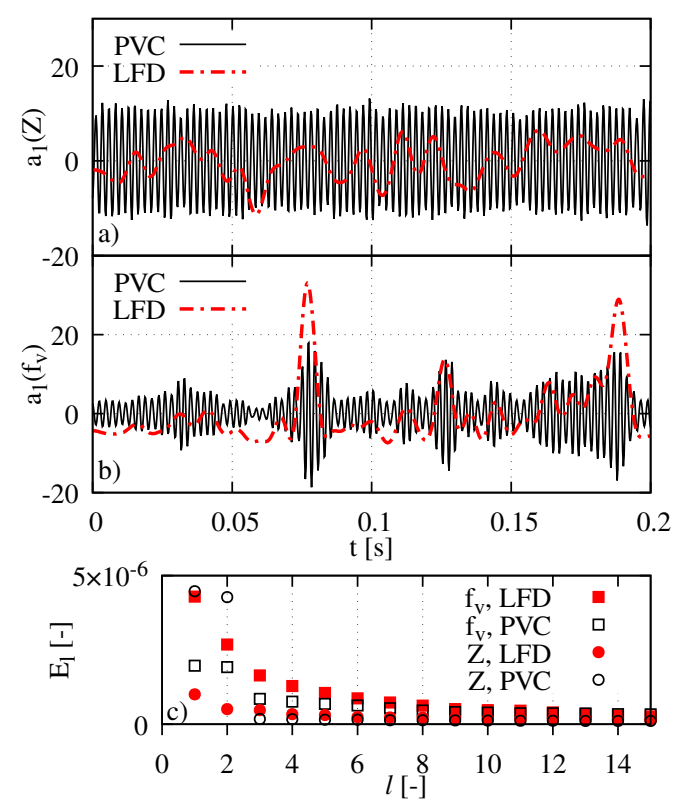

Figure 7: Time segment of LFD and PVC temporal coefficients for a) $\mathrm{Z}$ and b) $\mathrm{f}_{\mathrm{v}}$; c) Energy of the respective modes.

ior. The periodic PVC signal varies strongly in magnitude and interaction of the PVC and LFD modes is visible. As $a_{1, \mathrm{LFD}}$ rises, the amplitude of $a_{1, \mathrm{PVC}}$ rises, too, and vice versa. This shows that LFD do not only directly affect soot evolution but also influence the sensitivity of soot to PVC. Such differences in the sensitivities of $\mathrm{Z}$ and $\mathrm{f}_{\mathrm{v}}$ to PVC and LFD are also found in the energies of their respective modes. Figure $7 \mathrm{c}$ shows that, while the energy contained in the PVC modes of $\mathrm{Z}$ is much larger than in the LFD modes, the opposite holds for $f_{\mathrm{v}}$. It is clear that, unlike a steady quantity such as $\mathrm{Z}$ that is primarily affected by PVC dynamics, the highly intermittent soot evolution is strongly influenced by LFD.

The physical meaning of the most energetic soot mode $a_{1, \mathrm{LFD}}\left(f_{\mathrm{v}}\right)$ can be deduced from Fig. 8 where its temporal evolution is plotted along the soot 
volume in the combuster $\iiint\left(\mathrm{f}_{\mathrm{v}}\right) d V$. Even though the magnitude of both

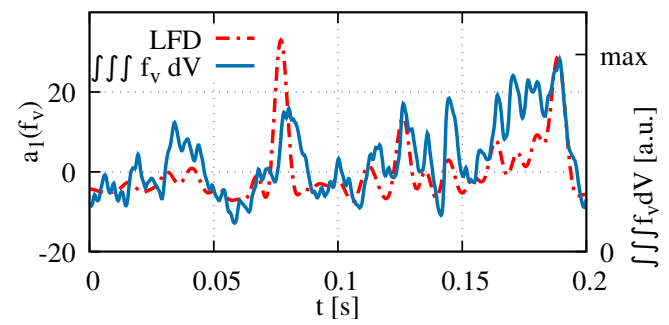

Figure 8: Time segment of LFD temporal coefficient for $\mathrm{a}_{1}\left(\mathrm{f}_{\mathrm{v}, \mathrm{LFD}}\right)$ and the soot volume in the combustor.

quantities differs in some positions, similar trends are clearly present. Hence, $a_{1, \mathrm{LFD}}\left(\mathrm{f}_{\mathrm{v}}\right)$ can be seen as a marker for the sooting behavior of the combustor. In order to assure, that other LFD modes of $f_{v}$ are less characteristic for the soot evolution, cross-correlations of the modes with the integrated soot volume fraction have been evaluated. It is found that the correlation for $a_{1, \mathrm{LFD}}\left(\mathrm{f}_{\mathrm{v}}\right)$ is 2.5 times higher than for any of the other modes (not shown), emphasizing its relevance.

To clarify how low-frequency dynamics in the flow field influences soot evolution, correlations among temporal coefficients of the LFD modes for $f_{v}, Z$ and $v_{\mathrm{x}}$ are analyzed. Since the LFD signals are non-periodic, the normalized cross-correlation coefficient $\mathrm{R}$ is used. As mentioned earlier, LFD dynamics do not appear as coherent structures and spread nearly evenly across multiple modes. This complicates the choice of modes for comparison. For demonstrative purposes, LFD modes of $f_{v}$ and $\mathrm{Z}$ exhibiting the strongest correlations with those of $\mathrm{v}_{\mathrm{x}}$ are shown here. However, similar behaviors have been observed for other LFD modes. The cross-correlations plotted in Fig. 9 reveal that $a_{1, \mathrm{LFD}}\left(\mathrm{f}_{\mathrm{v}}\right)$ and $a_{2, \mathrm{LFD}}\left(\mathrm{v}_{\mathrm{x}}\right)$ are not directly correlated. However, 


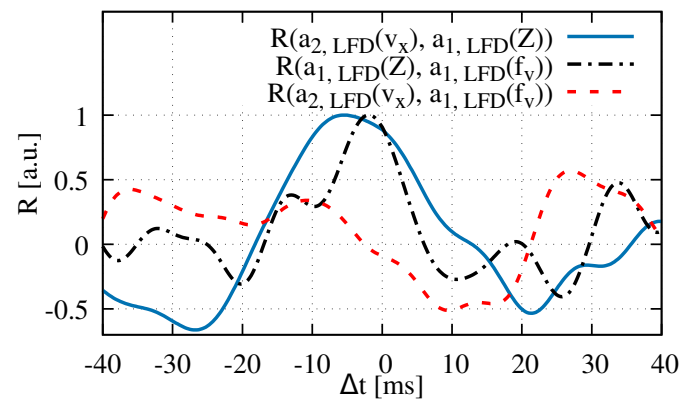

Figure 9: Normalized cross-correlation of LFD modes.

both $a_{1, \mathrm{LFD}}\left(\mathrm{f}_{\mathrm{v}}\right)$ and $a_{2, \mathrm{LFD}}\left(\mathrm{v}_{\mathrm{x}}\right)$ are correlated to $a_{1, \mathrm{LFD}}(\mathrm{Z})$, which is indicated by peaks in the vicinity of $\Delta \mathrm{t}=0 \mathrm{~s}$. Combined with the spatial distribution of $\phi_{2, \mathrm{LFD}}\left(\mathrm{v}_{\mathrm{x}}\right)$ presented in Fig. 4 , the mechanism of LFD influence on soot evolution can be described as the following: Alternations in recirculated gas mass flow and jet flapping of the secondary air jets $\left(a_{2, \mathrm{LFD}}\left(\mathrm{v}_{\mathrm{x}}\right)\right)$ cause a change in mixture fraction $\left(a_{1, \mathrm{LFD}}(\mathrm{Z})\right)$. $\mathrm{Z}$ in turn is correlated to the most energetic LFD mode of soot evolution $\left(a_{1, \mathrm{LFD}}\left(\mathrm{f}_{\mathrm{v}}\right)\right)$. Hence, low-frequency flow field dynamics have a major impact on soot evolution and intermittency in the aero-engine model combustor.

\section{Conclusion}

In this work, MRPOD is applied to LES data of a turbulent sooting flame inside an aero-engine model combustor. It is shown that the application of MRPOD is necessary to extract PVC related dynamics of the highly intermittent soot field. This was not possible with conventional POD. The analysis of the velocity field revealed the presence of two major types of dynamics in the combustor. Firstly, the PVC and its harmonics which mainly affect the region close to the primary injector. Secondly, low-frequency dynamics 
which influences the entire combustor. The latter is associated with a flapping of the secondary air jets, and a change of recirculated mass flow as well as a slow drift in the flow field near the primary injector. Both dynamics are found to affect soot evolution. The PVC acts on $f_{v}$ both by a displacement of soot filaments and by chemical reactions induced by mixing. However, soot precursors like PAHs as well as soot nucleation are more strongly affected by chemical reactions. The comparison of LFD and PVC dynamics reveals that

low-frequency dynamics have the major impact on soot evolution. These LFD of $f_{v}$ are found to be correlated to the velocity field via mixture fraction. Hence, jet flapping and the associated change in recirculated mass flow have a dominant impact on soot evolution. In conclusion, MRPOD proved to be a valuable tool to investigate the interaction of flow field dynamics and soot evolution in the aero-engine model combustor. This study highlights the potential of the method for analyzing intermittent quantities in datasets with a relatively small number of snapshots.

\section{Acknowledgments}

Funding from the European Union within the project SOPRANO (Soot Processes and Radiation in Aeronautical innovative combustion) Horizon 2020 Grant Agreement No. 690724 is gratefully acknowledged. The authors gratefully acknowledge the Gauss Centre for Supercomputing e.V. (www.gausscentre.eu) for funding this project by providing computing time through the John von Neumann Institute for Computing (NIC) on the GCS Supercomputer JUWELS at Jülich Supercomputing Centre (JSC). 


\section{References}

[1] K. P. Geigle, R. Hadef, W. Meier, Soot formation and flame characterization of an aero-engine model combustor burning ethylene at elevated pressure, Journal of Engineering for Gas Turbines and Power 136 (2014) 021505.

[2] C. Eberle, P. Gerlinger, K. P. Geigle, M. Aigner, Numerical investigation of transient soot evolution processes in an aero-engine model combustor, Combustion Science and Technology 187 (2015) 1841-1866.

[3] S. T. Chong, M. Hassanaly, H. Koo, M. E. Mueller, V. Raman, K. P. Geigle, Large-eddy simulation of pressure and dilution-jet effects on soot

formation in a model aircraft swirl combustor, Combustion and Flame 192 (2018) 452-472.

[4] M. Stöhr, K. P. Geigle, R. Hadef, I. Boxx, C. D. Carter, M. Grader, P. Gerlinger, Time-resolved study of transient soot formation in an aeroengine model combustor at elevated pressure, Proceedings of the Combustion Institute 37 (2019) 5421-5428.

[5] Z. Yin, M. Stöhr, Time-frequency localisation of intermittent dynamics in a bistable turbulent swirl flame, Journal of Fluid Mechanics 882 (2020) A30.

[6] K. Taira, S. L. Brunton, S. T. M. Dawson, C. W. Rowley, T. Colonius, B. J. McKeon, O. T. Schmidt, S. Gordeyev, V. Theofilis, L. S. Ukeiley, Modal analysis of fluid flows: An overview, AIAA Journal 55 (2017) 4013-4041. 
[7] P. J. Schmid, Dynamic mode decomposition of numerical and experimental data, Journal of Fluid Mechanics 656 (2010) 5-28.

[8] E. Kaiser, B. R. Noack, L. Cordier, A. Spohn, M. Segond, M. Abel, G. Daviller, J. Östh, S. Krajnović, R. K. Niven, et al., Cluster-based reduced-order modelling of a mixing layer, Journal of Fluid Mechanics 754 (2014) 365-414.

[9] S. Barwey, M. Hassanaly, Q. An, V. Raman, A. Steinberg, Experimental data-based reduced-order model for analysis and prediction of flame transition in gas turbine combustors, Combustion Theory and Modelling 23 (2019) 994-1020.

[10] F. Nicoud, F. Ducros, Subgrid-scale stress modelling based on the square of the velocity gradient tensor, Flow, Turbulence and Combustion 62 (1999) 183-200.

[11] J. Bardina, J. Ferziger, W. Reynolds, Improved subgrid-scale models for large-eddy simulation, Proc. 13th Fluid and Plasma Dynamics Conference (1980) AIAA 1980-1357.

[12] S. S. Girimaji, Assumed $\beta$-pdf model for turbulent mixing: Validation and extension to multiple scalar mixing, Combustion Science and Technology 78 (1991) 177-196.

[13] P. Gerlinger, Investigation of an assumed pdf approach for finite-rate chemistry, Combustion Science and Technology 175 (2003) 841-872.

[14] M. E. Mueller, H. Pitsch, Large-eddy simulation subfilter modeling of soot-turbulence interactions, Physics of Fluids 23 (2011) 115104. 
[15] N. Slavinskaya, P. Frank, A modelling study of aromatic soot precursors formation in laminar methane and ethene flames, Combustion and Flame 156 (2009) 1705-1722.

[16] T. Blacha, M. Di Domenico, P. Gerlinger, M. Aigner, Soot predictions in premixed and non-premixed laminar flames using a sectional approach for PAHs and soot, Combustion and Flame 159 (2012) 181-193.

[17] C. Eberle, P. Gerlinger, M. Aigner, A sectional PAH model with reversible PAH chemistry for CFD soot simulations, Combustion and Flame 179 (2017) 63-73.

[18] C. Eberle, P. M. Gerlinger, M. Aigner, Large-eddy simulations of a sooting lifted turbulent jet-flame, Proc. 55th AIAA Aerospace Sciences Meeting (2017) AIAA 2017-1785.

[19] M. Grader, C. Eberle, P. Gerlinger, M. Aigner, LES of a pressurized, sooting aero-engine model combustor at different equivalence ratios with a sectional approach for PAHs and soot, Proc. ASME Turbo Expo (2018) GT2018-75254.

[20] P. Nau, Z. Yin, K. P. Geigle, W. Meier, Wall temperature measurements at elevated pressures and high temperatures in sooting flames in a gas turbine model combustor, Applied Physics B 123 (2017) 279.

[21] L. Sirovich, Turbulence and the dynamics of coherent structures. i. coherent structures, Quarterly of Applied Mathematics 45 (1987) 561-571.

[22] I. Daubechies, Ten Lectures on Wavelets, Society for Industrial and Applied Mathematics, 1992. 
[23] M. Stöhr, R. Sadanandan, W. Meier, Phase-resolved characterization of vortex-flame interaction in a turbulent swirl flame, Experiments in Fluids 51 (2011) 1153-1167.

[24] Z. Yin, I. Boxx, W. Meier, Influence of self-sustained jet oscillation on a confined turbulent flame near lean blow-out, Proceedings of the Combustion Institute 36 (2017) 3773-3781.

[25] M. Stöhr, K. Oberleithner, M. Sieber, Z. Yin, W. Meier, Experimental study of transient mechanisms of bistable flame shape transitions in a swirl combustor, Journal of Engineering for Gas Turbines and Power 140 (2017) 011503. 